\title{
TECHNIQUE
}

\section{Prolonged monitoring of the upper gastrointestinal tract using echo planar magnetic resonance imaging}

\author{
D F Evans, G Lamont, M K Stehling, A M Blamire, P Gibbs, R Coxon, J D Hardcastle, \\ P Mansfield
}

\begin{abstract}
Upper gastrointestinal motility and transit has been studied in five human volunteers with the ultra high speed, magnetic resonance imaging (MRI) technique MBEST (Modulus Blipped Echo-planar Single pulse Technique), a variant of echo planar imaging. Snapshot images requiring a data acquisition time of only 64-128 msec allowed visualisation of peristalsis in the antrum and duodenum in real time, without motional image degradation, as would normally be seen using conventional MRI Gastroduodenal flow of the luminal contents was visualised using water as a contrast medium, with appropriate adjustment of the time constant (T2) weighting of the system. Rapid $(0.3 \mathrm{~Hz})$ imaging of gastroduodenal motility in both transverse and coronal planes was achieved by respiratory gating to the imaging frequency, allowing repetition rates of up to 20 frames per minute for 2-3 hours. Fast replay of stored images, to produce a 'movie loop', allowed identification of fasting motility patterns of the gastric antrum and proximal small intestine, with depiction of the phases of the migrating motor complex. Images of the fed patterns after the ingestion of a test meal showed good separation of solid and liquid particulate matter and mixing waves in the gastric body. The potential for quantitative measurements with this new imaging modality of the gastrointestinal tract is under development, which will allow us to measure transit and correlate this with motility data.

(Gut 1993; 34: 848-852)
\end{abstract}

In the last decade magnetic resonance imaging (MRI) has become an established diagnostic tool in clinical medicine and improvements in experimental technique and equipment are still continuing at a considerable pace.' Standard magnetic resonance imaging techniques require repeated sampling of the nuclear magnetic resonance signal in a series of experiments, which may take several minutes to complete ${ }^{23}$ and are therefore only suitable for the study of static organs. Imaging of organs that are characterised by periodic motion, such as respiration or the beating heart are more difficult to obtain and require synchronisation of the MRI scanner with, for example, the QRS signal of the ECG waveform. Abdominal imaging so far has been disappointing because of the aperiodic motion of the gut wall and its contents. This results in image artefacts and blurring of the spatial detail of the organ. ${ }^{4}$ Investigators have resorted to paralysing the smooth muscle with spasmolytics to obtain artefact free images of the gastrointestinal tract. This prevents, however, dynamic studies of peristalsis and motility patterns, an area of great interest at the present time.

Gastrointestinal tract imaging has been achieved using the ultrafast, echo-planar imaging, 5 variant technique MBEST (Modulus Blipped Echo-planar Single pulse Technique). ${ }^{67}$ MBEST produces a complete two dimensional image in acquisition times of either 64 or $128 \mathrm{~ms}$ for $128 \times 64$ or $128 \times 128$ pixel matrices respectively. These times are sufficiently short to freeze abdominal motion and thus overcome motional blurring. Additionally, rapid repetition of the experiment allows the production of abdominal movies without any harmful effects to the subjects. We have reported a series of experiments of short term gastrointestinal tract imaging using breathholding to overcome respiratory movement. ${ }^{8}$ This paper presents an improvement of this technique in which we have used voluntary, respiratory gating and have been able to obtain images for periods of 2-3 hours. Thus, for the first time, recordings of fasting and fed motility patterns of the upper gastrointestinal tract have been achieved.

\section{Methods}

\section{IMAGING PRINCIPLES}

Echo planar imaging is a real time magnetic resonance imaging method capable of producing snapshot images in times ranging from 64-128 ms. The details of the technique have been presented elsewhere ${ }^{5-7}$ but the main features are as follows. The patient is placed in a static magnetic field. In our case the field strength of the Nottingham home built imaging system utilises a 0.52 tesla superconductive magnet, corresponding to a resonance frequency for protons of $22 \mathrm{MHz}$. After an initial slice selection procedure, the nuclear magnetic resonance signal is subjected simultaneously to two modulated magnetic field gradients. One, $G_{y}$, is in the form of a bipolar trapezoidal modulation, the other, $G_{x}$, in the form of monopolar short 
duration blips. These two gradients effectively define the coordinate structure of the image in the $x-y$ plane. The strengths and modulation periods define the in-plane pixel resolution.

The effect of these gradients is to cause the nuclear magnetic resonance signal to form a series of spin echoes. These signals are sampled, Fourier transformed, and after data reordering, are presented to an image display device. The initial data sampling period is typically $128 \mathrm{~ms}$ for abdominal images. The Fourier transform times depend on the computer used, but in our case can take around $100 \mathrm{~ms}$. The display refresh time is around $80 \mathrm{~ms}$ which limits our real time frame rate to a maximum of three frames per second. In our current work, however, we run somewhat slower at about 1 frame $/ 2$ secs.

The MBEST technique is a variant of echoplanar imaging and currently produces images with an in plane spatial resolution of $3 \mathrm{~mm}$ for a $128 \times 64$ pixel matrix. The spatial resolution may be increased to $1.5 \times 3 \mathrm{~mm}$ using a $128 \times 128$ image pixel matrix but with some loss in signal intensity. The slice thickness was $1 \mathrm{~cm}$. MBEST images have an inherent contrast that discriminates against tissues with a short spin-spin relaxation time. This time constant (T2), characterises the duration of the nuclear magnetic resonance signal from water and depends on how water is bound in the tissue. Free water, as in cerebrospinal fluid and urine has a long T2 of up to several seconds, whereas water bound in protein, as in muscle tissue, has a shorter T2 of some $100 \mathrm{~ms}$. In MBEST, the main magnetic resonance signals (nuclear magnetic resonance spin echoes) occur midway through the imaging sequence, giving periods of $32 \mathrm{~ms}$ or $64 \mathrm{~ms}$ in the $128 \times 64$ or $128 \times 128$ experiments. During this time signals from tissue with a short $\mathrm{T} 2$, for example, the gut wall, can experience appreciable decay and thus appear dark. Free water - that is, luminal content, with a long T2, will appear as a bright image.

Image contrast also depends on the spin lattice relaxation time ( $\mathrm{T} 1$ ), which again is long for free water and shorter in tissue. As the T1 determines how long it takes for the magnetic resonance signal to recover after each image, a long $\mathrm{T} 1$ that is, 1-3 seconds as in water, determines the maximum image frequency achievable in continuous, long duration experiments. Chemical shift artefacts are excluded by selective presaturation of the undesired proton species (fat). Pure water images also help delineation of abdominal organs from mesenteric fat. ${ }^{59}$

The values of static field strength, gradient switching rates, and resonance frequency values all fall within the National Radiological Protection Board guidelines for clinical MRI. At $22 \mathrm{MHz}$ the mean resonance frequency power used during initial slice selection procedure is $5 \mathrm{~W}$ per pulse. This is equivalent to a specific absorbed power of $0.002 \mathrm{~W} / \mathrm{Kg}$ over the frame repetition period of $3 \mathrm{sec}$ per pulse.

SUBJECTS AND PROTOCOL

Three male and two female volunteers aged $20-43$ with no history of gastrointestinal diseases and in a healthy state, were studied. Subjects gave written informed consent after ensuring that they had no metal implants or foreign bodies (metal implants, excluding dental fillings, will degrade the potential images obtained using MRI). All subjects were given 11 of tap water before imaging, the water acting as a positive that is, bright contrast medium, to outline the gut lumen without affecting motility state. ${ }^{10}$

Images were obtained from all subjects lying supine, within the scanner, in the transverse or coronal planes at the level of the gastric antrum. The best image resolution was obtained from transverse images because of the orientation of the resonance frequency coils. We felt, however, that coronal imaging might give more detailed information regarding the anatomical detail of the antropyloric region and therefore attempted to image in the coronal plane using reorientation of the nuclear magnetic resonance signals by signal processing methods. As yet, coronal images are not of such high resolution as the transverse sequences. Examples of both imaging modalities are shown in the results for comparison.

\section{SCANNING PROCEDURE}

The MBEST technique, with real time data processing, takes only a fraction of a second to generate and display an image on the video screen, therefore correct positioning of the subject was achieved by simply moving him/her within the coil until the plane of interest was identified. In all subjects the following imaging technique was used. Subjects were instructed to breathe in synchrony to the imaging frequency of 20 frames per min. This was easily achieved as an audible 'ping' is produced by the scanner as each snapshot is taken. In this way a continuous recording of antroduodenal motility could be achieved.

We attempted to visualise the two major physiological states of upper gastrointestinal motility. The fasting state, which consists of a rhythmic cycle of contractile events occurring in the stomach and small intestine in the interdigestive state and termed the migrating motor complex." The migrating motor complex consists of a cyclical period of activity with a length of about 120 minutes in man. This period is divided into three distinct phases, phase I total quiescence $(30-80 \%$ of cycle), phase II intermittent contractions $(0-75 \%$ of cycle), and phase III, a short period (4-10 minutes) of intense contractions at the maximum contractile rate, determined by slow wave frequency (11-12 cycles per minute in the duodenum in man) and followed by a new phase I. These patterns of contractile activity occur in all healthy subjects and are normally recorded with tube mounted pressure sensors.

The fed state replaces fasting activity during ingestion of a nutrient meal and this motility state was also examined, using the MBEST scanning technique. After a variable period of fasting recording, depending on the assessment of the occurrence of a phase III, subjects were given an egg and mayonnaise sandwich, to stimulate the fed pattern. The meal was given with an additional volume of $500 \mathrm{ml}$ water and 

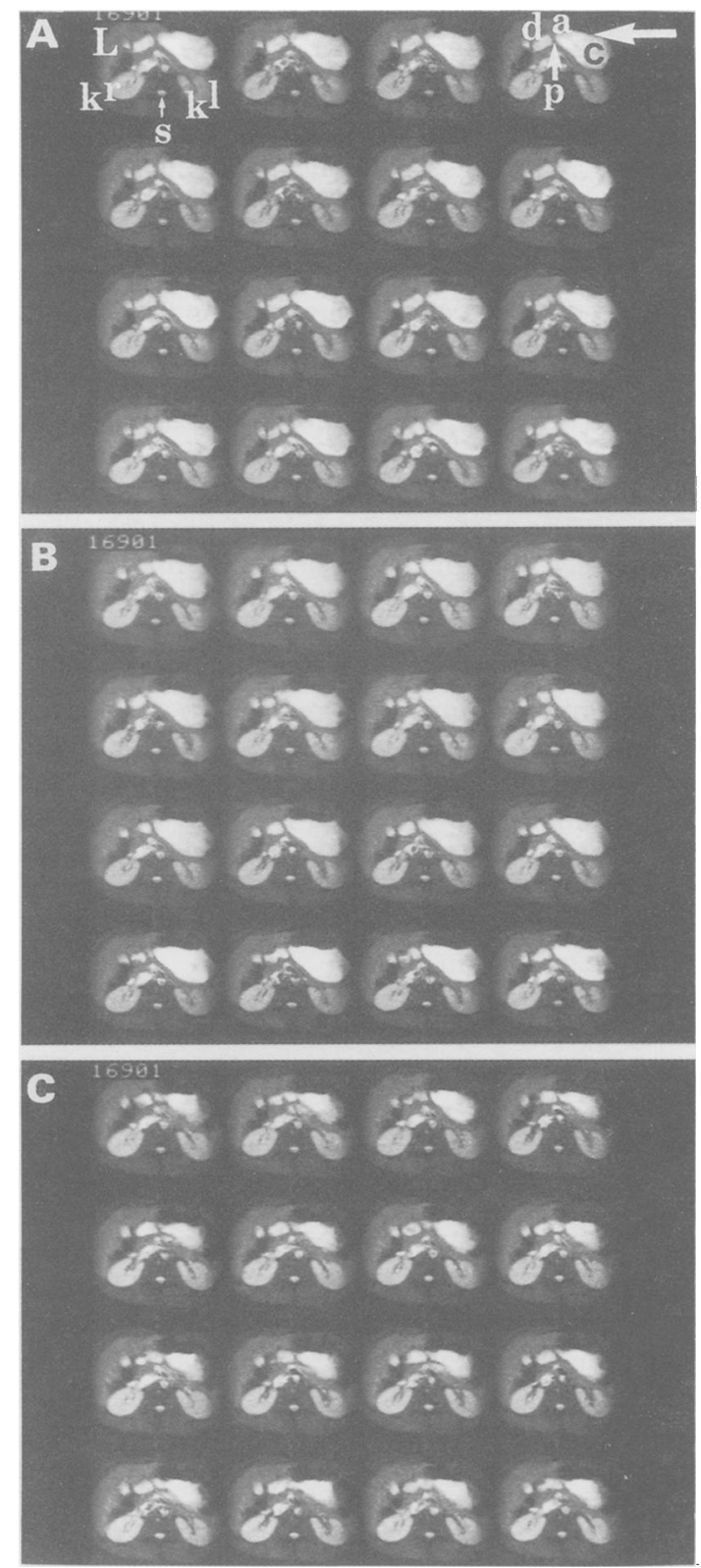

Figure 1: In this subject the respiratory gated images of the three phases of the migrating motor complex are illustrated. The corpus $(c)$, antrum $(a)$, pylorus $(p)$, duodenal bulb $(d)$, and fluid level (arrowed) can be seen in the transverse plane. (A) This sequence depicts quiescence in the gastric antrum and duodenum with no visible contractile activity. No movement of fluid is apparent. This is phase $I$. (B) This sequence with intermittent contractions in frames 6-10 and 13-16 shows vigorous contractile activity in the antroduodenal region. This is interspersed with quiescent frames and probably represents phase II $(C)$ Vigorous, rapid antroduodenal contractions in almost all frames together with progressive emptying of fluid from the stomach with regular 14 per minute contractions represent late phase II/phase III of the migrating motor complex.

imaging was continued for a further 40 minutes after the meal.

\section{DATA PRESENTATION AND ANALYSIS}

The two dimensional images were displayed at the time of acquisition and simultaneously stored into computer hard disc memory by an array processor. Each image occupied 16 Kbytes of computer storage, thus for a three hour study, a total of $57.6 \mathrm{M}$ bytes was required. Data were downloaded on to magnetic tape for long term storage.
After the experiment, magnetic resonance images were reconstructed by one dimensional Fourier transform and Fourier filtering techniques, including spatial correlation to reduce unwanted noise. ${ }^{12}$ Images could then be viewed as still sequences (Fig 1) or displayed as a continuous real or accelerated sequence or 'movie' loop, or parts, or of the whole study. Calculations of frequency and wave velocity of motility could be derived directly from the screen.

\section{Results}

\section{ANTRODUODENAL MOTILITY: THE MIGRATING} MOTOR COMPLEX

Figure 1 shows transverse images through the abdomen of a volunteer in the fasted state at the height of the gastric antrum. This series of frames was taken during a 90 minute period; the subject was given 11 of tap water to highlight the antrum and duodenum.

Sixteen, consecutive frames, viewed from top left to bottom right, imaged at $0 \cdot 3 \mathrm{~Hz}$ are shown in $\mathrm{A}, \mathrm{B}$, and $\mathrm{C}$. The transverse $1 \mathrm{~cm}$ slice is as viewed from below. The left and right kidneys are marked $\left(K^{l} \& K^{r}\right)$. The right lobe of the liver (L) and cerebrospinal fluid in the spinal cord (s) are also easily seen. Water, seen as a bright image, fills the stomach and delineates the corpus (c), the antrum (a), and the duodenal bulb (d). The pylorus is seen as an incisure (p), and shows closure of the viscus. A fluid level (arrowed), shows the presence of a gas bubble which appears black. Each image was comprised of $128 \times 128$ pixels and took $128 \mathrm{msec}$ to take. The images in Fig 1 were taken from sections of a movie loop with a total of 912 frames, the stills have been selected to illustrate the phases of the migrating motor complex.

Figure 1A represents phase I. This sequence shows no visible peristaltic activity, was present for the first 35 minutes of the study, and is typical of the pattern seen in conventional manometric recordings.

Figure 1B represents phase II of the migrating motor complex and depicts periods of intermittent antroduodenal activity interspersed with quiescent periods. The figure shows two such quiescent/active cycles. On viewing the whole sequence as a video movie the phasic pattern is more obvious. This pattern occupied 45 minutes of the total sequence which approximates to the phase II period in conventional studies. Swirls of activity, denoting movement of the gastric content can be seen in the fundus in some of the frames.

Figure $1 C$ represents the final 10 minutes of the fasting sequence and depicts the late phase II/ phase III of the migrating motor complex cycle. During the final five minutes of this sequence a period of intense activity was obvious and coincided with the emptying of water from the stomach and duodenal bulb. It was also associated with vigorous coordinated antroduodenal contractions. The movie loop of this sequence showed a reducing volume of fluid in the stomach when compared with Fig $1 \mathrm{~A}$ and also, as in Fig 1B 'swirls' of activity are apparent, 
Figure 2: These frames are coronal images of the stomach and duodenum and illustrate both morphology and function. The stomach is crescent shaped and the closed pylorus is clearly illustrated (arrow). Gastric mixing can be seen as changes of luminal density from frame to frame. $(A)$ Represents the fasted state. (B) Ingested meal particles can be clearly seen in the gastric body with some caudal movement being visible during the sequence.
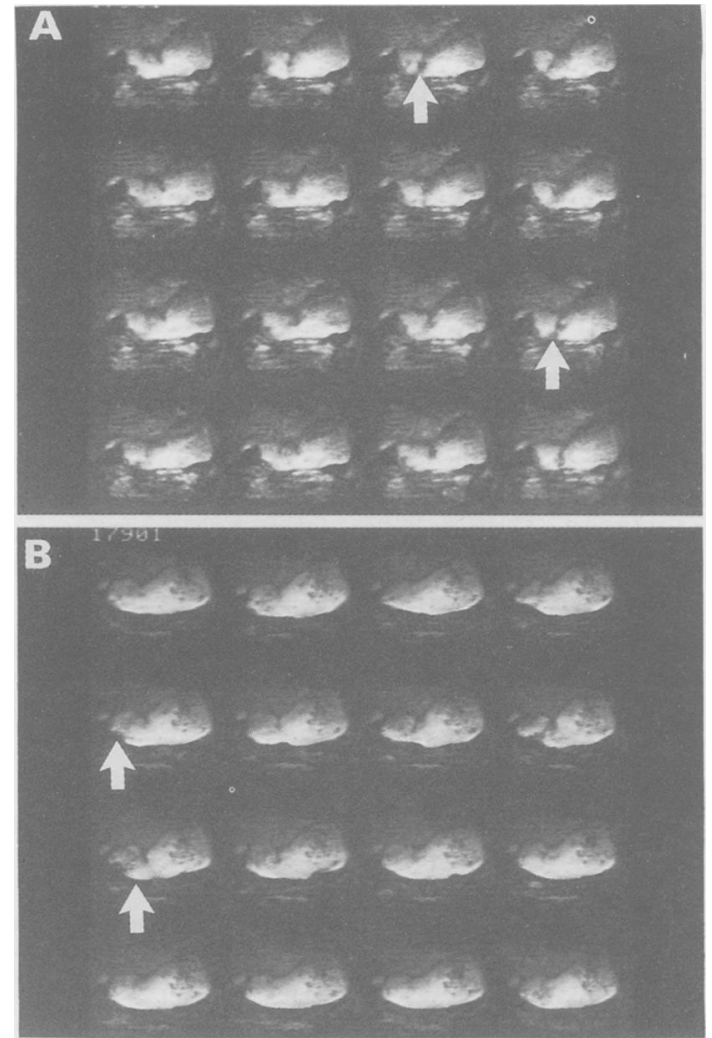

especially in the fundic region, denoting considerable movement of fluid and eddy currents as the contents are propelled towards the pylorus and on into the duodenum.

The frequency of peristalsis in the duodenum was calculated from this sequence at 14 contractions per minute. This value is similar to previously reported values for duodenal contractile frequency of 12 to 13 contractions ${ }^{10}$ and supports the proposal that these images are phase III like activity patterns.

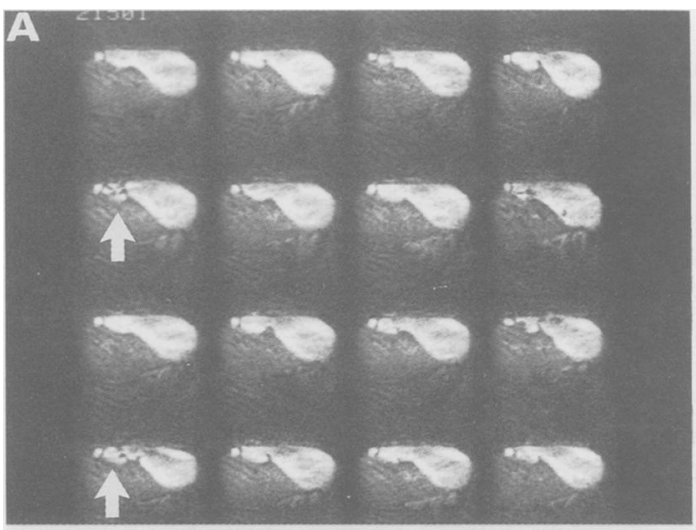

Figure 3: (A) In this female subject the stomach is pear shaped in these coronal views. The pylorus is well depicted in some frames (arrowed). Antral contractions can also be seen. (B) After ingestion of the test meal, stronger antral contractions can again be seen (frames 6 and 11) and the particulate matter of the sandwich is well visualised. In this case there is no apparent reduction in particle size or gastric emptying.
ANTRODUODENAL MOTILITY: FASTING AND FED PATTERNS

Figures 2 and 3 were taken from a series of images from a male (Fig 2) and female (Fig 3) volunteer. The images were taken in the coronal plane with a repetition time of 5 seconds and represent fasting (A) and fed (B) activity. Morphologically the stomach is similar to that seen with a conventional anterior/posterior view barium meal. Because coronal images are derived from the transverse plane by signal processing, the resolution is influenced by susceptibility artefacts. These can be seen as 'ripples' within the image and reduce the clarity in some frames.

Figure 2A depicts alternative frames from the 13 to the 17 minute of a 20 minute fasted sequence after preloading with 11 of tap water. The lumen of the stomach appears bright with a near homogeneous content because of the long T2 of the water. In this subject the stomach is crescent shaped and the pylorus can be seen as an incisure at its distal end (arrowed). Contractile changes in the stomach wall can be seen throughout the sequence, depicting mixing waves and fasting phasic contractions. Fast video replay of these frames illustrate the contractile activity more clearly than the freeze frames of this figure.

Figure 2B depicts a similar sequence after ingestion of the egg sandwich. The stomach now appears more distended when compared with the fasted sequence and powerful pyloric contraction can be seen (arrowed). This four frame sequence is equivalent to 20 seconds real time thus giving a contractile frequency of 3 per minute, the reported maximum rate in man. In the first frame, individual dark particles of the sandwich show clearly in the fundus and body. Later in the sequence the gastric contents take on a slightly more homogeneous quality, which may be caused by a reduction in particle size, although more prolonged imaging would be required to confirm this conclusively.

Figures $3 \mathrm{~A}$ and $3 \mathrm{~B}$ illustrate a similar sequence in the female subject. The stomach in this subject is pear shaped, with good resolution of the antrum and pylorus. Frames showing definite antropyloric contractions are arrowed. The fed sequence (Fig 3B) again shows marked antropyloric activity but little apparent particle reduction or gastric emptying of the meal.

\section{Discussion}

This paper has shown the feasibility of using echo-planar magnetic resonance imaging for prolonged imaging of upper gastrointestinal motility. The ability of the technique to simultaneously assess morphology and function, makes it an ideal medium for investigation of the physiology of the human gastrointestinal tract.

We have already shown that this type of imaging technique can overcome the problems of conventional MRI. ${ }^{8}$ With the addition of gated respiration and adjustment of the flip angle and relaxation time, together with reconstruction of longer imaging periods, we have been able to extend studies to facilitate the investigation of fasting periodic phenomena and some patterns of gastroduodenal fed activity. This had only been 
possible before using invasive manometric techniques or ionising radiation.

Limitations of this system at present are that subjects can only be studied in the supine position within the magnet, thus imposing constraints on both posture and movement. This may well influence gastrointestinal motility, as others have shown changes as a result of ambulation and stress. ${ }^{131+}$ Also, this technique requires the use of a contrast medium, in this case, tap water. Water is an ideal contrast medium as it does not abolish the fasting migrating motor complex, but it has a long $\mathrm{T} 1$ relaxation time of at least 3 seconds and that limits the imaging frequency of our system to 20 frames per minute. In future, experiments may be performed using Gadolinium DTPA, a paramagnetic contrast agent, which predominantly shortens the $\mathrm{T} 1$ relaxation time to allow faster imaging. This substance, however, is not yet available in an oral form. The use of such substances would enhance the resolution of the images by increasing the signal to noise ratio between tissue and contrast media and allow for a greater imaging frequency because of the shorter relaxation time. This would facilitate the calculation of peristaltic velocity and the speed of contractility of gut smooth muscle with high temporal resolution.

The ability to visualise the stomach in the fed state may help to increase our knowledge of gastric function, especially in tracing the passage of gastric chyme during the initial stages of digestion. This may help in the investigation of conditions where gastroparesis is a clinical problem. Because MRI is particularly well developed to differentiate between lipid and water, with further development, it may be possible to simultaneously measure transit and absorption of different nutrients in the gut, an area of measurement at present not possible with existing methods. Further stages of investigation using this technique will be to simultaneously measure gastrointestinal motility using conventional manometric methods and to compare this with echo-planar imaging as a validation of the imaging technique.

Rapid development is taking place in MRI technology. Recently, another ultrafast MRI technique has been introduced under the name of 'snapshot' or turboFLASH. ${ }^{15}$ This technique, which derives its name from the Fast, Low angle, single-Shot method, provides images in about $500 \mathrm{~ms}$, fast enough to resolve peristaltic motion. 'Snapshot' flash may be superior to echoplanar imaging in some instances, especially where appreciable amounts of bowel gas are present. This is because considerable local signal loss can occur with echo-planar imaging where gas tissue interfaces cause susceptibility artefacts which may obscure adjacent tissues, such as the bowel wall. 'Snapshot' flash is insensitive to this effect and allows imaging of gas filled bowel without loss of detail. ${ }^{16}$ At present, however, echo-planar imaging provides better spatial and temporal resolution as well as a better signal to noise ratio than 'snapshot' flash. It also allows three dimensional imaging without time penalty which is advantageous for dynamic studies of motility and transit in the gastrointestinal tract. If susceptibility artefacts with echo-planar imaging can be overcome, the method will be even better for non-invasive imaging of the gut and more sophisticated imaging will be possible.

At present echo-planar imaging is only available in a few research centres worldwide. Several major manufacturers of magnetic resonance systems, however, are currently developing echo planar imaging on their systems: as an add-on feature, echo-planar imaging is now commercially available. (Advanced NMR Systems, Mass, USA.) With more efficient magnetic gradient coils and more sophisticated, cost effective computer power, echo planar imaging will be an available option on most conventional MRI scanners in the next couple of years. Routine imaging of gastrointestinal motility could then become as commonplace as conventional MRI.

The Medical Research Council, the Department of Health of Great Britain, and the British Heart Foundation, all financially support the Echo-Planar imaging programme in Nottingham. The Deutsche Forschungsgemeinschaft, the Science and Engineering Research Council, Analogic Inc, and Oxford Magnet Technology are acknowledged for contributions towards salaries Technology are acknowledged for contributions towards salaries
and hardware. We are grateful to Dr Roger Ordidge for his assisand hardware. We are grateful to Dr Roger Ordidge for his assis-
tance in various stages of the project and Mr Mark Simms and Dr Penny Gowland for assistance with data processing and photography.

1 Mansfield $P$. Imaging by nuclear magnetic resonance. $\mathcal{F}$ Phys $E$ Sci Instrum 1988; 21: 18-30.

2 Kumar A, Welti D, Ernst RR. NMR Fourier Zeugmatography. F Mag Res 1975; 18: 69-83.

3 Edelstein WA, Hutchinson JMS, Johnson G, Redpath T. Spin-warp NMR imaging and applications to human wholebody imaging. Phys Med Biol 1980; 25: 751-6.

4 Wood ML, Henkelman RM. MR image artefacts from periodic motion. Med Phys 1985; 12: 143-51.

5 Mansfield P. Multi-planar image formation using NMR spin echoes. Fournal of Physics C: Solid State Physics 1977; 10: L55-8.

6 Howseman AM, Stehling MK, Chapman B, Coxon R, Turner $\mathrm{R}$, Ordidge RJ, et al. Improvements in snap-shot NMR imaging. Br $\mathcal{f}$ Radiol 1988; 61: 822-8.

7 Stehling MK, Howseman AM, Ordidge RJ, Chapman B, Turner R, Coxon R, et al. Whole body echo-planar imaging at 0.5 Tesla. Radiology 1989; 170: 257-63.

8 Stehling MK, Evans DF, Lamont G, Ordidge RJ, Howseman AM, Chapman B, et al. Gastrointestinal tract: dynamic MR studies using echo-planar imaging. Radiology 1989; 171: $41-6$.

9 Ordidge RJ, Howseman A, Coxon R, Turner R, Chapman B, Glover P, et al. Snapshot imaging at 0.5 Tesla using Echo Planar techniques. Magn Reson Med 1989; 10: 227-40.

10 Thompson DG, Wingate DL. Effects of osmoreceptor stimulation on human duodenal motor activity. Gut 1988; 29: $173-80$.

11 Itoh Z, Aizawa I, Sekiguchi T. The interdigestive migrating motor complex and its significance in man. Clin Gastroenterol 1982; 11: 497-521.

12 Doyle M, Mansfield P. Real-time movie image enhancement in NMR. Fournal of Physics E: Scientific Instruments 1986; 19: 439-44.

13 Kumar D, Wingate DL, Ruckebusch Y. Circadian variation in the propagation velocity of the migrating motor complex. Gastroenterology 1986; 91: 926-30.

14 Valori RM, Kumar D, Wingate DL. Effects of different types of stress and of prokinetic agents on control of the fasting
motor complex in humans. Gastroenterology 1986; 90: motor compl.

15 Haase A. Snapshot FLASH MRI. Applications to T1, T2 and chemical shift imaging. Magn Reson Med 1990; 13: 77-89. 16 Hagspiel KD, Stehling MK, von Schulthess GK. Gastrointestinal tract: dynamic MR studies with fast gradient echo imaging. Proceedings of the European Society of Magnetic Resonance in Medicine and Biology. Zurich, 1991: 325. 